\title{
Design and Implementation of Spatial Correlation-Based Clustering for Multiuser MISO-NOMA Systems
}

\author{
Caner Goztepe $^{\circledR}$, Berna Ozbek ${ }^{\circledR}$, Senior Member, IEEE, and Gunes Karabulut Kurt ${ }^{\circledR}$, Senior Member, IEEE
}

\begin{abstract}
In this letter, we propose a novel user clustering algorithm for downlink multiuser multiple-input single-output (MISO) non-orthogonal multiple access (NOMA) systems along with its implementation in a real-time testing environment. The proposed method selects the clusters by considering users' spatial channel properties against the generated orthogonal directions. This is then followed by power allocation and zero-forcing precoding steps to mitigate the interference between the selected clusters. Performance comparisons are provided in terms of both real-time tests and simulations. It is demonstrated that a notable improvement in capacity and reliability can be obtained through the proposed approach in multiuser MISO-NOMA systems with reduced complexity.
\end{abstract}

Index Terms-Non-orthogonal multiple access (NOMA), multiuser multiple-input single-output (MU MISO), spatial correlation-based clustering, software-defined radio.

\section{INTRODUCTION}

A $S$ THE number of devices and the user expectations increase, the need for a higher spectral efficiency becomes more critical in communication systems. Different methods are being developed by the research community to meet users' expectations. One of these methods is the joint use of multiple-input single-output (MISO) and the power domain non-orthogonal multiple access (NOMA), where the same frequency band is used in the same time period by distinct users in order to increase the number of users that establish a reliable transmission and a high sum data rate [1], [2].

In [3], multiuser NOMA has been studied by jointly using the zero-forcing $(\mathrm{ZF})$ precoding and power allocation. For MISO-NOMA systems, the optimal beamforming solution for minimizing transmission power of all the users with quality of service (QoS) constraints has been examined in [4], [5]. Although the dirty paper coding (DPC) based approach maximizes the capacity region it is difficult to implement due to its high complexity [4]. In our work, the zero-forcing (ZF) precoding, is employed as a lower complexity solution.

For MISO-NOMA with ZF precoding, it has been shown that clustering slightly correlated users are more likely to suppress the inter-cluster interference to increase the spectral

Manuscript received August 22, 2020; accepted August 31, 2020. Date of publication September 3, 2020; date of current version January 8, 2021. This research was supported by TUBITAK under Grant 1160179. The associate editor coordinating the review of this letter and approving it for publication was P. Yang. (Corresponding author: Caner Goztepe.)

Caner Goztepe and Gunes Karabulut Kurt are with the Department of Electronics and Communication Engineering, Istanbul Technical University, 34469 İstanbul, Turkey (e-mail: goztepe@itu.edu.tr; gkurt@itu.edu.tr).

Berna Ozbek is with the Department of Electrical-Electronics Engineering, Izmir Institute of Technology, 35430 İzmir, Turkey (e-mail: bernaozbek@iyte.edu.tr).

Digital Object Identifier 10.1109/LCOMM.2020.3021466 efficiency. In order to reduce the correlation between the clusters, there are different user selection methods in the literature [6]. Semi-orthogonal user selection (SUS) methods, which are more suitable approach for real-time applications due to their flexibility, are among the most frequently utilized user selection algorithms [7]-[10]. [7] highlighted the potential of selecting a small number of users in terms of sum rate improvement. The impact of a limited feedback has been considered in [8]. The authors of [9] investigated the possible diversity gain, again in presence of limited a feedback. The user selection with a widely linear transmit precoding in multiuser communication has been presented in [10]. The sorting algorithm in [11] and the SUS algorithm with user matching technique [12] have been also adopted for MISO-NOMA. In addition to that, an improved version of the SUS algorithm has been examined in [13] to further increase the sum data rate in a defined scenario with specified regions for the weak and strong users, which may not be practically applicable in all deployment scenarios.

In the existing studies, the performance evaluations are frequently investigated in terms of capacity, while leaving the error performances unexamined. Recently, error performance of general NOMA systems have been examined. Closed-form error performance analysis and real-time testing environment performance have been examined for BPSK modulation in the basic NOMA system in [14]. However, the downlink multiuser MISO-NOMA approaches in the literature are difficult to apply in real-time systems in highly dense environment, because of their complexity.

As it is extremely important to demonstrate the practical applicability and the achievable performance in real-time systems, in this letter, a low complexity user clustering approach is proposed in dense user deployment scenarios for downlink multiuser MISO-NOMA systems. The performance evaluation of the proposed method is provided in terms of both sum data rate and error performance. The contributions are given as follows:

- For the first time in the literature, the traditional multiuser MISO-NOMA system is designed in a real-time testing environment by using software-defined radios (SDR). Its performance is tested under different conditions.

- A novel user clustering algorithm based on spatial correlation against the generated orthogonal directions is proposed in order to improve system overall performance. The proposed approach provides a significant gain with a reduced complexity for a high number of users.

- The error performances of the traditional and the proposed schemes are investigated in a comparative manner, 


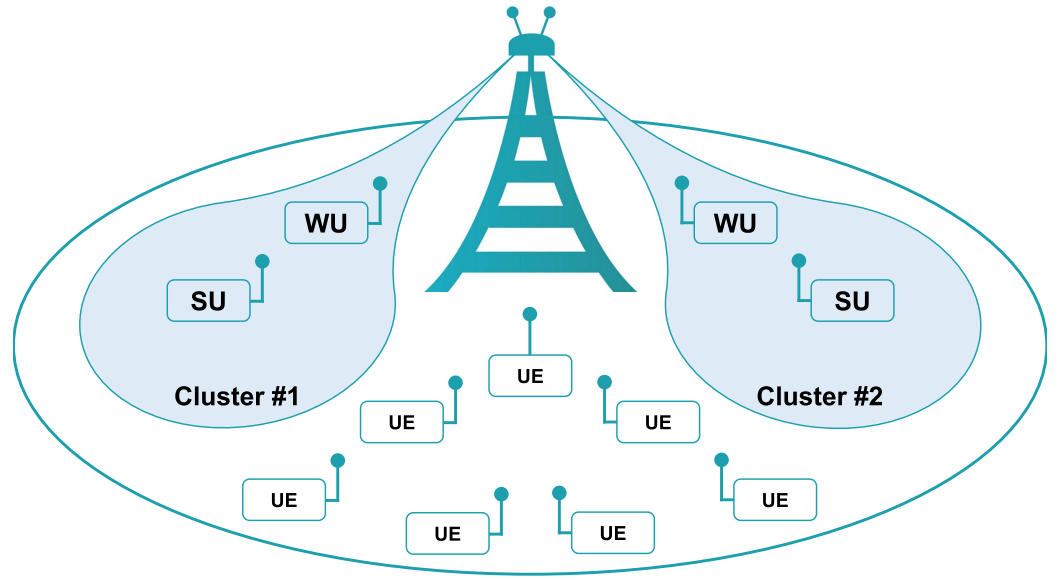

(a)

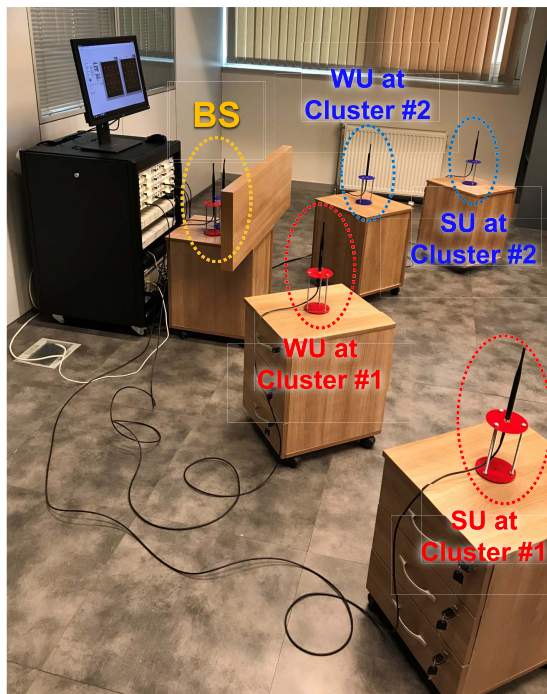

(b)

Fig. 1. (a) The proposed scheme for the downlink multiuser MISO-NOMA including strong user (SU) and weak user (WU) for each cluster. (b) The real-time test-bed including 2 clusters and 4 users.

through both simulation and test studies. The designed real-time SDR based test-bed includes a base station (BS) with 2 transmit antennas and 4 user equipment (UE) devices equipped with a single receive antenna.

This letter is organized as follows. Section II describes the multiuser MISO-NOMA system model. Section III introduces the proposed algorithm and provides its performance based on the sum data rate. The real-time test-bed is described in Section IV. The test and simulation results are discussed in Section V. The conclusions are drawn in Section VI.

\section{SySTEM MOdEL}

As illustrated in Figure 1a, we consider multiuser MISO systems combined with power domain NOMA including $N_{t}$ antennas at the transmitter and $K$ single antenna users in a cell. The total number of clusters is denoted by $C$ and each cluster accommodates $U$ users. Since we consider dense deployment scenarios, the number of users that establish reliable transmission is high. The number of clusters is selected as the number of transmit antennas while considering more than one user at each cluster through NOMA technique. In this work, we select $U$ users among $K$ users for each cluster through the proposed user clustering algorithm as detailed in Section III.

The transmitted symbol belonging to the $u^{\text {th }}$ user at the $c^{\text {th }}$ cluster is denoted by $x_{u, c}$, where $1 \leq u \leq U$ and $1 \leq c \leq C$. Then, the transmitted NOMA symbol belonging to the $c^{\text {th }}$ cluster is defined by,

$$
x_{c}=\sum_{u=1}^{U} \sqrt{\alpha_{u, c}} x_{u, c} ; \quad \text { with } \sum_{u=1}^{U} \alpha_{u, c}=1 ;
$$

where $\alpha_{u, c}$ is the power coefficient for the $u^{\text {th }}$ user at the $c^{\text {th }}$ cluster. At each cluster, the power coefficients are determined based on the users' channel gains while an equal power allocation is performed between clusters.

The channel vector of size $N_{t} \times 1$ between the transmitter and the $u^{\text {th }}$ user at the $c^{\text {th }}$ cluster is denoted by $\mathbf{h}_{u, c}=d_{u, c}^{-\alpha} \mathbf{f}_{u, c}$ where $d_{u, c}$ is the distance between the corresponding user and the BS, $\alpha$ is the exponent of path loss, $\mathbf{f}_{u, c}$ is the complex channel coefficient for Rayleigh fading.

For each cluster, the relation $\left\|\mathbf{h}_{1, c}\right\|>\left\|\mathbf{h}_{2, c}\right\|>\ldots>$ $\left\|\mathbf{h}_{U, c}\right\|$ is maintained, where the user 1 and the user $U$ are respectively the strongest and weakest users in each cluster. In order to mitigate inter-cluster interference in the multiuser MISO-NOMA system, we perform ZF precoding by considering either the weakest user (WU) or the strongest user (SU) from each cluster. Under consideration of the strongest users from all clusters, the $C \times N_{t}$ dimensional channel matrix is represented by $\mathbf{H}$ :

$$
\mathbf{H}=\left[\mathbf{h}_{1,1} \mathbf{h}_{1,2} \cdots \mathbf{h}_{1, C}\right]^{T} .
$$

The $\mathrm{ZF}$ precoding matrix is determined by:

$$
\mathbf{W}^{\prime}=\mathbf{H}^{H}\left(\mathbf{H H}^{H}\right)^{-1},
$$

Then, the normalized ZF precoding matrix of the dimension of $N_{t} \times C$ is $\mathbf{W}=\left[\mathbf{w}_{1} \mathbf{w}_{2} \cdots \mathbf{w}_{C}\right]$, where $\mathbf{W}=\frac{\mathbf{W}^{\prime}}{\left\|\mathbf{W}^{\prime}\right\|}$.

The $N_{t} \times 1$ transmitted NOMA vector is given by:

$$
\mathbf{x}=\sum_{c=1}^{C} \mathbf{w}_{c} x_{c} .
$$

The received signal of the $u^{\text {th }}$ user at the $c^{\text {th }}$ cluster is:

$$
\begin{aligned}
y_{u, c}= & \left(\mathbf{h}_{u, c}\right)^{H} \mathbf{w}_{c} \sqrt{\alpha_{u, c}} x_{u, c} \\
& +\left(\mathbf{h}_{u, c}\right)^{H}\left(\mathbf{w}_{c} \sum_{j=1}^{u-1} \sqrt{\alpha_{j, c}} x_{j, c}+\sum_{i=1 ; i \neq c}^{C} \mathbf{w}_{i} x_{i}\right) \\
& +n_{u, c},
\end{aligned}
$$

where $n_{u, c}$ denotes the additive white Gaussian noise (AWGN) component with zero mean and variance $\sigma_{n}^{2}$ for the $u^{\text {th }}$ user at the $c^{\text {th }}$ cluster.

In Eq. (5), the first term is the desired signal, while the second term includes the interference coming from the 
other clusters and users. In order to detect the symbols at the user side, either maximum likelihood (ML) or successive interference cancellation (SIC) can be used [15]. By using the ZF precoding method applied to the strongest user, the sum data rate of the multiuser MISO-NOMA system with SIC can be calculated as follows [16]:

$$
R_{\text {sum }}=\sum_{c=1}^{C} \sum_{u=1}^{U} \mathbb{E}\left\{\log _{2}\left(1+\gamma_{u, c}\right)\right\}
$$

where $\gamma_{u, c}$ is the signal to interference and noise ratio (SINR) and calculated by:

$$
\gamma_{u, c}=\frac{\alpha_{u, c}\left|\mathbf{h}_{u, c}^{H} \mathbf{w}_{c}\right|^{2}}{\underbrace{\sum_{j=1}^{u-1} \alpha_{j, c}\left|\mathbf{h}_{u, c}^{H} \mathbf{w}_{c}\right|^{2}}_{\text {Inter-user interference }}+\underbrace{\sum_{i=1, i \neq c}^{C}\left|\mathbf{h}_{u, c}^{H} \mathbf{w}_{i}\right|^{2}}_{\text {Inter-cluster interference }}+\sigma_{n}^{2}} .
$$

\section{The Proposed User Clustering Algorithm}

While the multiuser MISO-NOMA system includes $K$ users, it only serves $U \times C$ users. Here, we propose an efficient user clustering methods based on spatial correlation against the generated orthogonal directions to improve overall system performance. Although several techniques have been examined for clustering the users in the literature as in [13], the proposed approach is more suitable for real-time applications, due to its reduced complexity and high flexibility.

First, $C$ complex random orthonormal vectors as $\boldsymbol{\theta}_{c} \in \mathbb{C}^{N_{t} \times 1}, c=1, \ldots, C$ are generated which correspond to the predefined clusters. For each user, the spatial correlation based on each predefined cluster is measured by using chordal distance:

$$
d^{2}\left(\overline{\mathbf{g}}_{k}, \boldsymbol{\theta}_{c}\right)=1-\left|\overline{\mathbf{g}}_{k}^{H} \boldsymbol{\theta}_{c}\right|^{2} ; \quad c=1, \ldots, C,
$$

where $\overline{\mathbf{g}}_{k}=\frac{\mathbf{h}_{k}}{\left\|\mathbf{h}_{k}\right\|}$ is the channel direction information (CDI) of the $k^{\text {th }}$ user.

Using the chordal distance metric, the $k^{\text {th }}$ user is assigned into the $c^{\text {th }}$ predefined cluster when the following criterion is satisfied:

$$
d^{2}\left(\overline{\mathbf{g}}_{k}, \boldsymbol{\theta}_{c}\right) \leq \epsilon,
$$

where $\epsilon$ is the predefined threshold value for $0 \leq \epsilon \leq 1$. As the chordal distance measures distances between subspaces of Euclidean $N_{t}$-dimensional space, it serves as an indicator of the orthogonality between the users' channel vectors, directly controlling the inter-cluster interference. The case of $\epsilon=1$ refers to the traditional multiuser MISO-NOMA system [11], where any user assignment is not applied.

In the proposed clustering algorithm, only the users which satisfy the spatial correlation metric in Eq. (9) are the candidates for the user selection at the BS. Then, only these users are considered to assign the predefined clusters based on their channel quality information (CQI). The user that has the maximum $\mathrm{CQI}$ is assigned as the $\mathrm{SU}$ and the one that has the minimum CQI and higher than a given threshold is selected as the WU in each cluster.

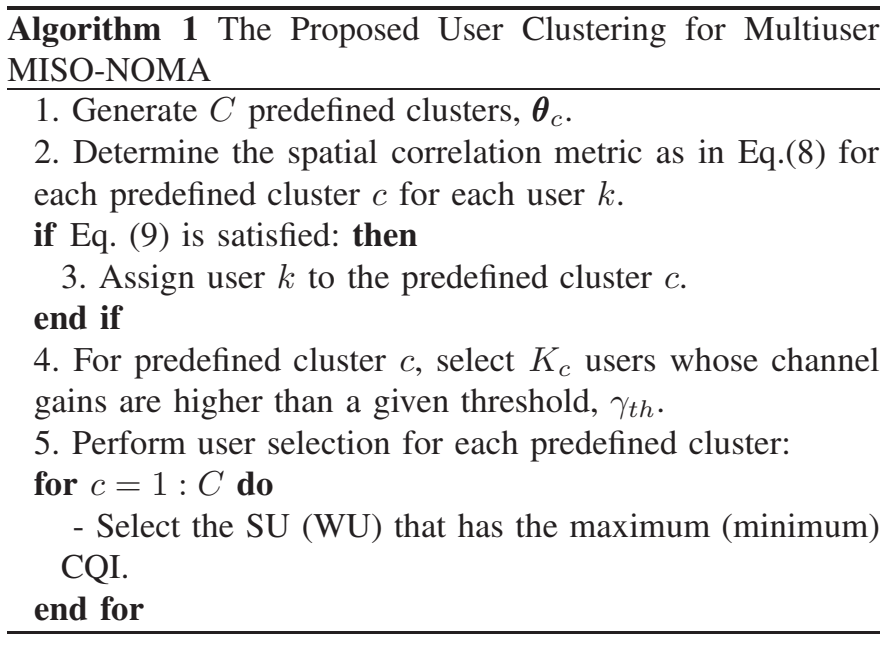

The proposed approach is detailed in Algorithm 1. After selecting the users through the Algorithm 1, the transmit vector is obtained by applying Eq. (1)-(4). In accordance with the practical applicability goal, the complexity of the proposed clustering algorithm is reduced since the number of users per cluster is significantly reduced through the spatial domain criterion for the downlink multiuser MISO-NOMA system in highly dense environments.

The complexity analysis of the proposed algorithm can be made based on the flop count calculations. Flop counts in each step of the proposed algorithm are $C, C K\left(6 N_{t}+4\right), C K$, $\sum_{c=1}^{C} K_{c}$ and $\sum_{c=1}^{C}\left(K_{c}-1\right)$ for steps 1 to 5 in Algorithm 1, where $K_{c}$ refers to the number of users obtained as a result of step 3 for cluster $c$, respectively. For $2 \leq C \leq N_{t}, 2 \leq U \leq$ $K_{c} \leq K$ and $U C \leq K$, the upper bound of the flop count of the proposed algorithm can be given by:

$$
\varphi \leq C+C K\left(6 N_{t}+4\right)+C K+\sum_{c=1}^{C} K_{c}+\sum_{c=1}^{C}\left(K_{c}-1\right),
$$

which can be shown to be:

$$
\varphi \leq 6 C K N_{t}+5 C K+2 \sum_{c=1}^{C} K_{c}=\mathcal{O}\left(C K N_{t}\right),
$$

where $\varphi$ denotes the flop count of the proposed algorithm. The proposed algorithm is less complex than the user selection algorithms that make use of channel sorting as in [11].

\section{A. Results on Sum Data Rate}

The performance results on the sum data rate are provided based on Eq. (3)-(6) for the case of uniformly distributed users whose average SNRs are between $0 \mathrm{~dB}$ to $30 \mathrm{~dB}$. SIC is used at the receivers. The power allocation coefficients are set to $1 / 5$ and $4 / 5$ for the $\mathrm{SU}$ and $\mathrm{WU}$, respectively. We select the threshold values as $\epsilon=0.25$ and $\gamma_{t h}=10 \mathrm{~dB}$ to keep the complexity low while having a good sum data rate performance. In the proposed algorithm, the average number of users per cluster is calculated as $\epsilon \times K$. As a result, instead of all users, we only consider $25 \%$ of users while performing user selection, which reduces the complexity of user-cluster algorithm significantly. 


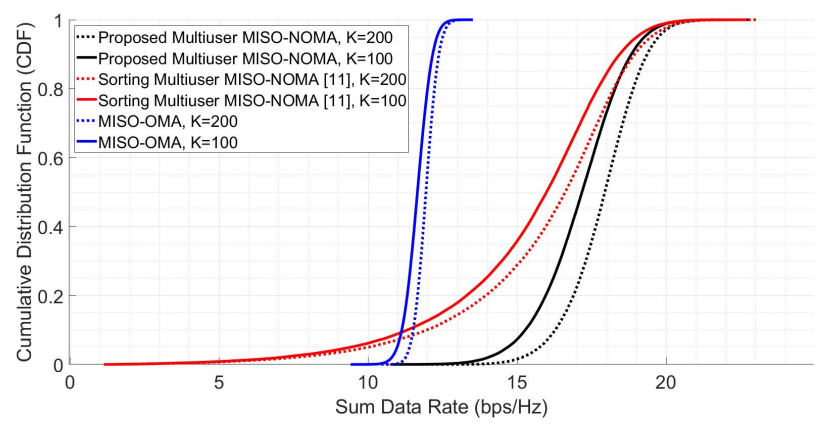

Fig. 2. Comparison of sum data rates of the considered approaches.

In Figure 2, the sum data rate of the proposed multiuser MISO-NOMA is compared to MISO-orthogonal multiple access (OMA) and traditional multiuser MISO-NOMA given in [11]. The MISO-OMA selects the $N_{t}$ best users in terms of CQI and then transmit them in orthogonal time slots. The proposed user clustering provides an improvement of about $1.5 \mathrm{bps} / \mathrm{Hz}$ when compared to that of the traditional multiuser MISO-NOMA having much less complexity and provides an average gain of $6 \mathrm{bps} / \mathrm{Hz}$ compared to the MISO-OMA. Besides, when the number of users increases in the system, the proposed method provides an improvement in the sum data rate with a lower complexity since it considers much less number of users in the selection process.

\section{Software-Defined Radio Test-Bed}

The multiuser MISO-NOMA real-time test system, in which the proposed method is examined, is given in Figure 1b. Three USRP-2943Rs are used as SDR nodes, each with two independent RF chains. One of them is operated as a transmitter with two antennas of the base station, and the others are operated as receivers of 4 different users in two different clusters. Users in the cluster are located 1 and 2 meters away from the transmitter as the strong and the weak user of that cluster, respectively. The angle between the two clusters is set at 90 degrees. The nodes are physically separated using 5 meter RF cables. SDR management is provided by LabVIEW programming software on the host computer, which is used for the configuration, control and data collection aspects of the transmitter and receivers. The real-time test system is operated at $2.45 \mathrm{GHz}$ frequency band with $1 \mathrm{MHz}$ bandwidth.

In order for real-time test systems to work properly, a robust synchronization should be ensured between the transmitter and receiver using coarse and fine synchronization methods. In the designed test-bed, NI CDA-2990 8-channel clock distribution source is used to provide coarse synchronization. To maintain fine synchronization, the sample timing offset (STO) and the carrier frequency offset (CFO) between the transmitter and receivers should be estimated. There are five data symbols and one pilot symbol in each fixed period. In addition, a pilot frame, consisting of 12 fixed periods placed at the beginning of the data frame, is used at each of the four receivers for STO and CFO estimation with the approaches given in [17], and then compensated digitally. BPSK modulation is used for pilot symbols. Pilot symbols are also used for SNR estimation and channel estimation for four users. Channel coefficients are estimated by the least square method, which is often preferred in communication systems due to its simplicity. In order to remove the channel effects from the received signal, the obtained channel coefficients are used in the $\mathrm{ZF}$ equalizer in each receiver. Each user in the designed system knows only the power allocation coefficients and the related cluster information. In the designed test-bed, a silicon and wood based obstacle is placed in front of the transmitter in order to prevent line-of-sight (LoS) between the transmitter and receiver. The Rayleigh fading channel characteristic used in the simulation environment is obtained in the real-time testing environment with this physical configuration. To make a fair comparison, average channel gains in the test-bed are measured and scaled with receivers gain.

\section{Simulation and Test Results}

The performance of the proposed method in the multiuser MISO-NOMA systems is evaluated through measurements and simulations. 4-QAM modulation is used with a transmission rate of 8 Mbps. As shown in Figure 1a, two different clusters with two users in each cluster are considered.

The impact of precoding process is analyzed separately for the WU (the first user of its cluster) and the SU (the second user of its cluster). The SU is configured to have a $10 \mathrm{~dB}$ higher SNR value than the WU in each cluster in both the simulation environment and the real-time testing environment. The power coefficients are kept constant in each cluster and selected as $\phi=\frac{\sqrt{\alpha_{1, c}}}{\sqrt{\alpha_{2, c}}}=0.125$ and $\phi=0.25$ in different tests. In the simulation environment, the threshold values for proposed method in $K=100$ users are $\epsilon=0.10$ and $\epsilon=0.25$. However, real-time tests are performed for only $\epsilon=0.25$.

There are 4 different users in the testing environment and tests are performed for 11 different SNR values between $10 \mathrm{~dB}$ and $30 \mathrm{~dB}$ with $2 \mathrm{~dB}$ intervals. According to the results, 4 users, who are divided into 2 different clusters in the testing environment, cannot handle the proposed method's selection criterion in each time point according to their channel status. Therefore, 4 users determined from $K=100$ different users in the simulations in the same time period and 4 users satisfying the proposed method's criterion in different time periods in the testing environment are evaluated as an equivalent case.

Figure 3 shows the error results under different conditions, both in simulations and real-time tests. RT abbreviation in the plots denotes the real-time environment. Since the power allocated for both clusters is equal, the error performances of the SU and the WU in the clusters are obtained to be equal. For this reason, only the users' comparisons are included in the result graphs without specifying the cluster. The SNR of the SU is $10 \mathrm{~dB}$ higher than the WU in each plot. For $\epsilon=1$, the proposed method is not performed in the simulation environment and 4 users are randomly selected among $K=100$ users. In a real-time testing environment, $10^{9}$ samples per user are examined for each case and $1.69 \times 10^{6}$ out of $10^{9}$ samples provide the $\epsilon=0.25$ criterion. Therefore $1.69 \times 10^{6}$ samples can be considered as the samples of the proposed method. There is no restriction for $\epsilon=1$, so each sample is used for error calculation. 


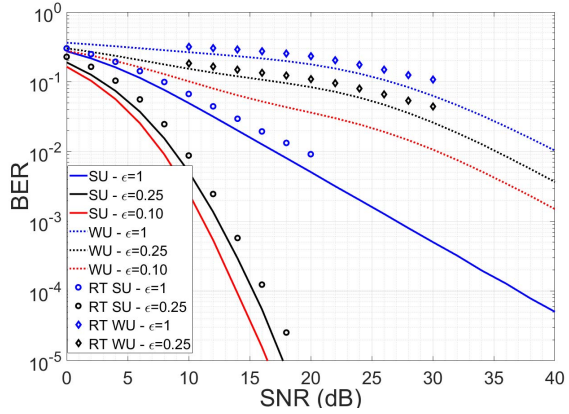

(a)

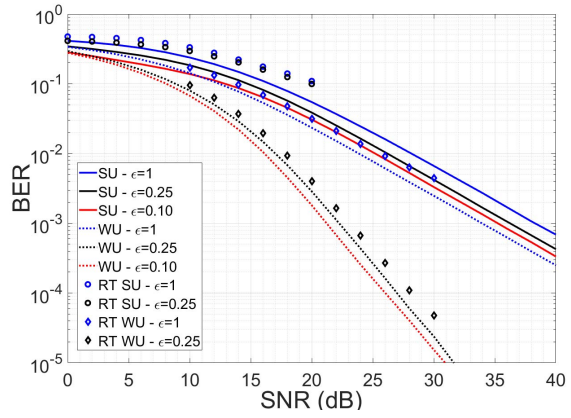

(b)

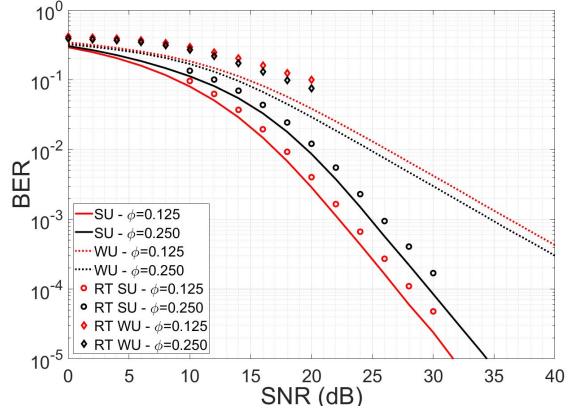

(c)

Fig. 3. (a) SU-based precoding at $\phi=0.125$. (b) WU-based precoding at $\phi=0.125$. (c) Comparison of SU-based and WU-based precoding at $\epsilon=0.25$.

In Figure $3 a$ and Figure $3 b, \phi=0.125$ is determined and the results of the systems that are precoded according to the SU and WU are shown, respectively. It is observed that the user selection method to be used in the precoding process is an important criterion for users' error performances. The precoding process has improved the SU's performance in each cluster considerably. As a result of the precoding process made according to the WU, the WU error performance exceeds the SU's error performance. The error performances of the SU and WU converge and a fair system in terms of error performance of users is established. Figure $3 \mathrm{a}$ and Figure $3 \mathrm{~b}$ show that the proposed method improves the error performance.

Figure $3 \mathrm{c}$ includes the precoding according to the WU and the power allocation coefficients are $\phi=0.125$ and $\phi=0.250$ for $\epsilon=0.25$. Differentiation of power allocation coefficients also changes the error performance. As a result of the increase of $\phi$, the SU can achieve 1-2 dB SNR gain and the WU observe approximately 2-3 dB SNR loss at the error value of $10^{-3}$. Different power allocation coefficients lead to different error performance among users in the system.

As shown in Figure 3, the use of the proposed method significantly improves the error performance of all users. The system operates more efficiently by reducing the threshold value. Due to the estimation methods used in the real-time testing environment, 1-3 dB SNR loss is observed with respect to the simulation results. As expected in the real-time testing environment, becoming distant from the simulation curve starts and an error floor occurs, specifically after $24 \mathrm{~dB}$ SNR. The results in the testing environment and the simulation results prove the success of the application of the proposed method in the multiuser MISO-NOMA system.

\section{CONCLUSION}

In this study, a multiuser MISO-NOMA system has been implemented in a real-time testing environment using SDR nodes for the first time in the literature. We have also proposed a spatial correlation-based clustering algorithm to increase the sum data rate and reliability. The performance of the proposed user clustering algorithm has been evaluated in terms of the average capacity and the error rates. Error rates are provided via simulation and real-time test results. It has been demonstrated that both the capacity and the error performance can be improved through the proposed method in the multiuser MISO-NOMA systems.

\section{REFERENCES}

[1] M. F. Hanif, Z. Ding, T. Ratnarajah, and G. K. Karagiannidis, "A minorization-maximization method for optimizing sum rate in the downlink of non-orthogonal multiple access systems," IEEE Trans. Signal Process., vol. 64, no. 1, pp. 76-88, Jan. 2016.

[2] C. Chen, W. Cai, X. Cheng, L. Yang, and Y. Jin, "Low complexity beamforming and user selection schemes for 5G MIMO-NOMA systems," IEEE J. Sel. Areas Commun., vol. 35, no. 12, pp. 2708-2722, Dec. 2017.

[3] B. Kimy et al., "Non-orthogonal multiple access in a downlink multiuser beamforming system," in Proc. IEEE Mil. Commun. Conf. (MILCOM), Nov. 2013, pp. 1278-1283.

[4] Z. Chen, Z. Ding, P. Xu, and X. Dai, "Optimal precoding for a QoS optimization problem in two-user MISO-NOMA downlink," IEEE Commun. Lett., vol. 20, no. 6, pp. 1263-1266, Jun. 2016.

[5] J. Zhu, J. Wang, Y. Huang, K. Navaie, Z. Ding, and L. Yang, "On optimal beamforming design for downlink MISO NOMA systems," IEEE Trans. Veh. Technol., vol. 69, no. 3, pp. 3008-3020, Mar. 2020.

[6] M. Shipon Ali, H. Tabassum, and E. Hossain, "Dynamic user clustering and power allocation for uplink and downlink non-orthogonal multiple access (NOMA) systems," IEEE Access, vol. 4, pp. 6325-6343, 2016.

[7] A. Tomasoni, G. Caire, M. Ferrari, and S. Bellini, "On the selection of semi-orthogonal users for zero-forcing beamforming," in Proc. IEEE Int. Symp. Inf. Theory, Jun. 2009, pp. 1100-1104.

[8] W. Ni, Z. Chen, H. Suzuki, and I. B. Collings, "On the performance of semi-orthogonal user selection with limited feedback," IEEE Commun. Lett., vol. 15, no. 12, pp. 1359-1361, Dec. 2011.

[9] M. Min, "On achievable multiuser diversity gain of limited-feedbackbased block diagonalization in interference-limited MIMO broadcast channels," IEEE Commun. Lett., vol. 23, no. 10, pp. 1717-1720, Oct. 2019.

[10] M. Bavand and S. D. Blostein, "User selection and multiuser widely linear precoding for one-dimensional signalling," IEEE Trans. Veh. Technol., vol. 67, no. 12, pp. 11642-11653, Dec. 2018.

[11] H. Zhang, D.-K. Zhang, W.-X. Meng, and C. Li, "User pairing algorithm with SIC in non-orthogonal multiple access system," in Proc. IEEE Int. Conf. Commun. (ICC), May 2016, pp. 1-6.

[12] S. Liu, C. Zhang, and G. Lyu, "User selection and power schedule for downlink non-orthogonal multiple access (NOMA) system," in Proc. IEEE Int. Conf. Commun. Workshop (ICCW), Jun. 2015, pp. 2561-2565.

[13] Y. N. Ahmed, "A novel scheduling technique for NOMA in 5G wireless communication systems," in Proc. 12th German Microw. Conf. (GeMiC), Mar. 2019, pp. 59-62.

[14] M. Aldababsa, C. Goztepe, G. K. Kurt, and O. Kucur, "Bit error rate for NOMA network," IEEE Commun. Lett., vol. 24, no. 6, pp. 1188-1191, Jun. 2020.

[15] M. S. Ali, E. Hossain, and D. I. Kim, "Non-orthogonal multiple access (NOMA) for downlink multiuser MIMO systems: User clustering, beamforming, and power allocation," IEEE Access, vol. 5, pp. 565-577, 2017.

[16] S. H. Amin, A. H. Mehana, S. S. Soliman, and Y. A. Fahmy, "User capacity in downlink MISO-NOMA systems," in Proc. IEEE Global Commun. Conf. (GLOBECOM), Dec. 2018, pp. 1-7.

[17] D. Oh, S. Kim, S.-H. Yoon, and J.-W. Chong, "Two-dimensional ESPRIT-like shift-invariant TOA estimation algorithm using multi-band chirp signals robust to carrier frequency offset," IEEE Trans. Wireless Commun., vol. 12, no. 7, pp. 3130-3139, Jul. 2013. 